\title{
Evaluación de los Procesos Software de una Organización Enfocando en la Disminución de la Resistencia al Cambio
}

\author{
Muñoz Mirna $^{1, *}$, Mejia Jezreel ${ }^{1}$, Calvo-Manzano Jose A. ${ }^{2}$, Cuevas Gonzalo², San Feliu Tomás ${ }^{2}$ \\ ${ }^{1}$ Centro de Investigación en Matemáticas Av. Universidad no 222, 98068 Zacatecas, México \\ ${ }^{2}$ Departamento Lenguajes y Sistemas Informáticos e Ingeniería de Software Universidad Politécnica de Madrid, Facultad de Informática \\ 28660 Boadilla del Monte, Madrid, España
}

\begin{abstract}
Resumen En este artículo se describe un método para la evaluación del rendimiento de los procesos software de una organización de desarrollo de software utilizando técnicas alternativas a los cuestionarios (utilizados actualmente como técnica principal para evaluar el rendimiento de los procesos software en las organizaciones de desarrollo software). La importancia de la evaluación de procesos de una organización radica en qué, si es realizada de manera correcta, esta actividad permite identificar las oportunidades de mejora y dirigir el esfuerzo de la mejora hacia los procesos software que necesitan ser mejorados para alcanzar los objetivos del negocio establecidos por la alta dirección con la finalidad de generar ventajas competitivas respecto de sus competidores y garantizar su permanencia en el mercado.
\end{abstract}

Palabras Clave Evaluación de Procesos Software, Rendimiento de Procesos Software, Mejora de Procesos Software, Objetivos del Negocio

\section{Introducción}

La calidad de los productos software depende en gran medida de los procesos utilizados para su creación[1]. En este contexto, mejorar los procesos utilizados por las organizaciones para el desarrollo de software es clave para obtener productos con la calidad esperada. Por lo tanto, se considera a la mejora de procesos software, como el camino más viable y lógico para que una organización genere ventajas estratégicas respecto a sus competidores y, como resultado, sea una empresa exitosa en la industria del software[2],[3],[4]. Esta es la principal razón por la que, la industria del software está cada vez más interesada por la mejora de procesos software (SPI, Software Process Improvement, por sus siglas en inglés).

Sin embargo, aún cuando muchas organizaciones están motivadas para implementar mejoras en sus procesos software, pocas son las que tienen el conocimiento para realizar este tipo de actividades[5].

Esta falta de conocimiento da como resultado que las mejoras en los procesos software no sean implementadas con éxito o tengan un éxito limitado. Además, se refleja en los esfuerzos dedicados a la mejora, ya que los implicados resultan en la mayoría de las veces frustrados, agotados y más convencidos que nunca de seguir realizando su trabajo como lo venían haciendo aún cuando no obtengan los

* Corresponding author:

mirna.munoz@cimat.mx (Muñoz Mirna)

Published online at http://journal.sapub.org/computer

Copyright (C) 2012 Scientific \& Academic Publishing. All Rights Reserved resultados esperados[5],[6].

Al respecto, autores como[7],[8],[9] han identificado que la evaluación de los procesos es un elemento clave para lograr la implementación exitosa de mejoras en los procesos software.

Desafortunadamente, la mayoría de las veces las evaluaciones de software son vistas como una amenaza, ya que el personal las percibe como una evaluación a su trabajo, por lo tanto, surge la resistencia al responder los cuestionarios utilizados durante las evaluaciones o éstos son respondidos con información muy alejada de la realidad.

El resultado de lo anterior es que el esfuerzo de mejora no se dirige adecuadamente, por lo tanto, los procesos obtenidos son ineficientes o extraños a quien los usa en la organización[10].

Como consecuencia, frecuentemente se observan dos situaciones o incluso ambas, por un lado, la resistencia a la implementación de mejoras en los procesos software incrementa y, por otro lado, la mejora de procesos no tiene los resultados esperados debido a que las carencias identificadas no reflejan las necesidades de mejora de los objetivos del negocio establecidos por la alta dirección.

El objetivo de este artículo es presentar un método que permite una evaluación interna y rápida del rendimiento de los procesos software de una organización de desarrollo de software enfocando en dos elementos clave: mejores prácticas de la organización y objetivos del negocio, permitiendo llevar a cabo evaluaciones rápidas y frecuentes del rendimiento de sus procesos, utilizando pocos recursos, y obteniendo como resultado una base sólida que resalta las necesidades de los objetivos del negocio establecidos de la 
organización.

Este artículo está estructurado como sigue: la sección 2, introduce a los conceptos básicos; la sección 3, muestra los antecedentes de la creación del método; la sección 4, presenta el método de evaluación; la sección 5, muestra los resultados de la aplicación del método; y la sección 6, presenta las conclusiones.

Tabla 1. Instituciones y modelos y/o estándares propuestos

\begin{tabular}{|c|c|}
\hline Institución & Modelos / estándares \\
\hline & Capability Maturity Model and \\
Instituto de Ingeniería del & Integration for Development \\
Software (SEI) & (CMMI-DEV)[13] \\
& Team Software Process (TSP)[14] \\
\hline Project Management & Project Management Body of \\
Institute (PMI) & Knowledge (PMBOK)[15] \\
\hline $\begin{array}{c}\text { Organización Internacional } \\
\text { para la Estandarización } \\
\text { (ISO) }\end{array}$ & ISO/IEC15504 Information \\
technology-Process assessment[16] \\
ISO/IEC 12207-2008[17]
\end{tabular}

\section{Conceptos Básicos}

\subsection{Mejores Prácticas}

Una mejor práctica puede ser una práctica técnica o de gestión, que ha demostrado una mejora sobre uno o más aspectos cómo: productividad, coste, calendario, calidad o satisfacción de usuario[11].

La importancia de las mejores prácticas, ha traído como consecuencia qué, instituciones de prestigio se enfoquen en el estudio de mejores prácticas, desarrollando modelos y estándares de referencia que contienen un conjunto de mejores prácticas que tienen una funcionalidad probada[12]. A continuación en la Tabla 1 se listan las principales organizaciones y sus modelos y estándares propuestos.

\subsection{Objetivos del Negocio e Indicadores}

Las organizaciones deben tener sus objetivos del negocio como principal referencia en la implementación de mejoras de procesos software. Para esta investigación los conceptos de objetivos del negocio e indicadores se listan a continuación:

- Objetivos del negocio: son objetivos establecidos por la alta dirección para garantizar su continuidad en el mercado y mejorar factores como rentabilidad, mercado y otros a ser logrados para el éxito de la organización[13]. Algunos ejemplos de objetivos del negocio son: reducción del tiempo del ciclo de desarrollo, reducción del número solicitudes de cambio durante la fase de integración, incrementar la satisfacción del cliente.

- Indicadores del negocio: son palabras que apoyan a la organización a especificar las medidas necesarias para obtener información acerca de los objetivos del negocio[18]. Algunos ejemplos de indicadores son: número de clientes, plazos de entrega citados, tiempo de desarrollo del ciclo de vida del producto, actas de arranque.

\section{Antecedentes}

\subsection{Metodología MIGME-RRC}

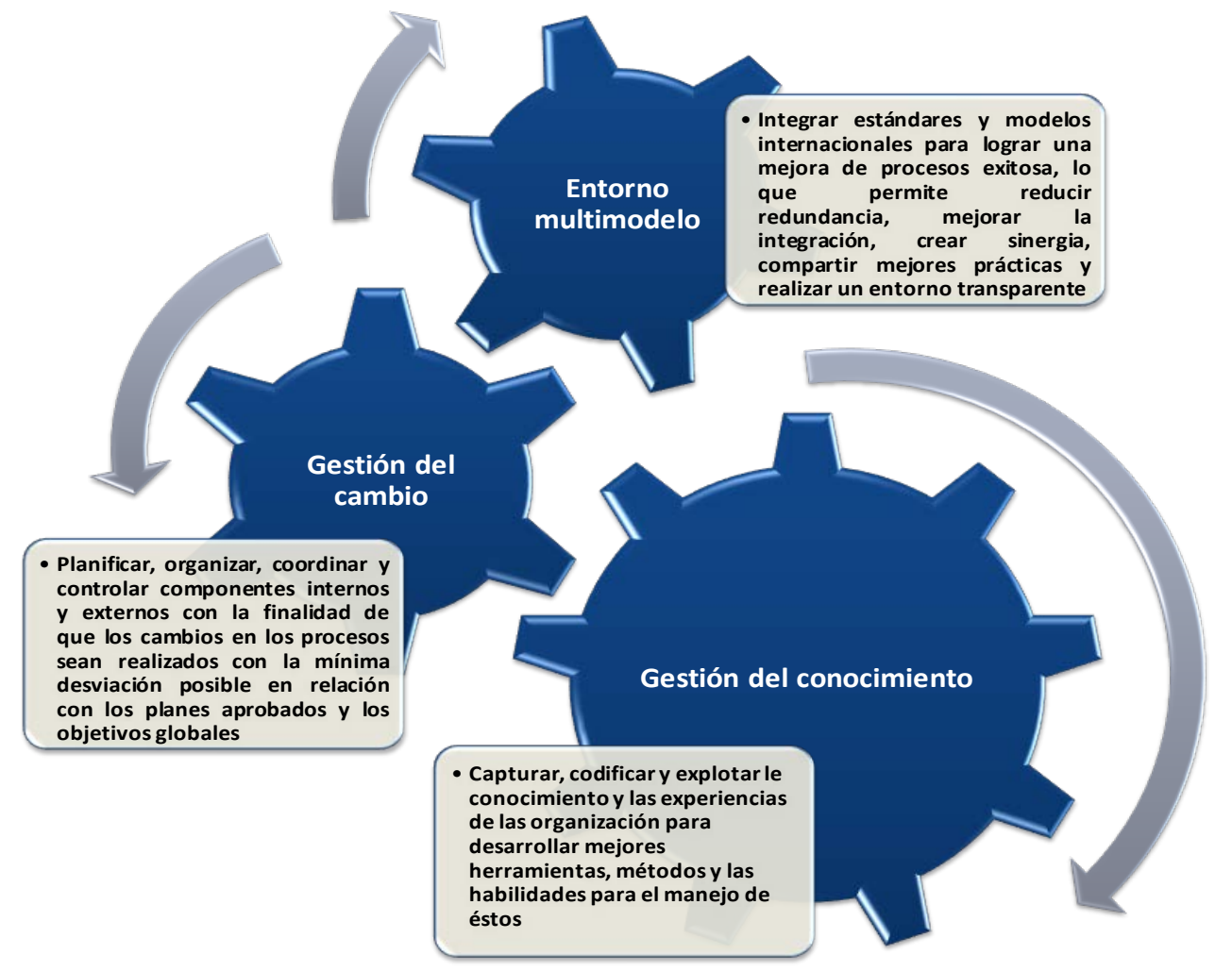

Figura 1. Conocimiento base de MIGME-RRC 
El método de evaluación propuesto en este artículo, fue desarrollado como parte de una metodología realizada como trabajo de tesis doctoral. La metodología desarrollada permite la implementación gradual y continua de mejoras en los procesos software de una organización, enfocándose en la disminución de la resistencia al cambio. La metodología ha sido llamada MIGME-RRC[19].

Cabe resaltar que para el desarrollo de la metodología MIGME-RRC fueron utilizados principios de la gestión del cambio, la gestión del conocimiento y entorno multimodelo. A continuación, en la Figura 1 se muestra brevemente el conocimiento tomado como base de cada una de éstas áreas.

Además, el desarrollo de la metodología, fue supervisado y apoyado por tres expertos en mejora de procesos de la Universidad Politécnica de Madrid y un gerente senior de la empresa everis consulting.

Como lo muestra la Figura 2, la metodología MIGME-RRC está compuesta de cuatro fases, las cuales se describen brevemente a continuación:

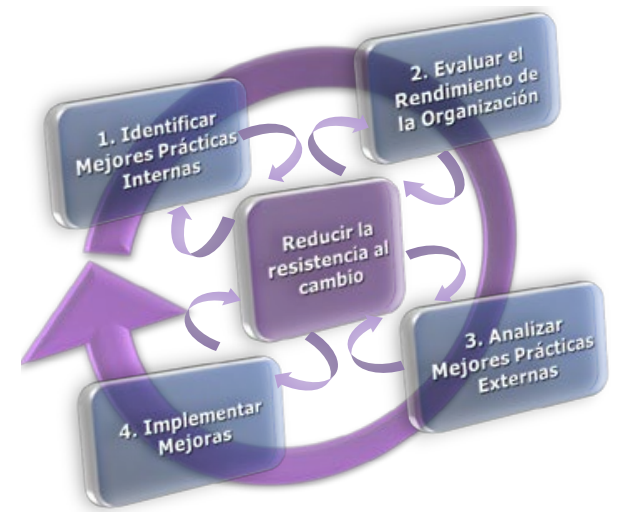

Figura 2. Metodología MIGME-RRC

1) Identificar mejores prácticas internas: actividades enfocadas en: la extracción del conocimiento tácito de la organización, y el análisis de la documentación formal de los procesos tal que se permita la identificación de las mejores prácticas de la organización, ayudando a entender la forma de trabajo de la organización.

2) Evaluar el rendimiento de la organización: actividades enfocadas a establecer el rendimiento de los procesos de la organización a través del análisis de cobertura de las mejores prácticas de la organización, los indicadores relacionados con los objetivos del negocio y los objetivos del negocio en sí mismos.

3) Analizar mejores prácticas externas: actividades enfocadas a establecer un entorno multimodelo que se utilizará como referencia para la selección de las mejores prácticas externas que mejor se ajusten a la forma de trabajo de la organización y hagan los procesos más eficientes.

4) Implementar mejoras: actividades enfocadas al diseño de los nuevos procesos a través de la integración de las mejores prácticas internas de la organización y las mejores prácticas externas seleccionadas con un entorno multimodelo

\subsection{Origen del Método de Evaluación}

El método de evaluación, por lo tanto, desarrollado como la segunda fase de la metodología MIGME-RRC, se basa en dos aspectos:

1) las necesidades y experiencias pasadas de la organización $y$,

2) los objetivos del negocio

El propósito del método de evaluación es determinar el rendimiento de los procesos software de una organización enfocando en las mejores prácticas reales llevadas a cabo en la organización y sus objetivos del negocio establecidos.

El método de evaluación propone realizar la evaluación interna de procesos de manera alternativa a la tradicionalmente utilizada por las organizaciones, como a continuación se describe:

1. Se realiza un análisis de cobertura "(bottom-up) de abajo- a arriba” entre las mejores prácticas, los indicadores y los objetivos del negocio.

2. Se realiza un análisis de cumplimiento entre los indicadores y objetivos del negocio.

3. Se realiza un análisis de priorización de los indicadores.

Mediante esta forma de evaluar los procesos software de una organización, se reduce la resistencia que normalmente surge con la aplicación de cuestionarios.

Además, de acuerdo a Pino et al.[7], las evaluaciones rápidas internas de procesos software son consideradas un elemento clave para el éxito en la implementación de mejoras de procesos software, debido a que pueden ser realizadas con frecuencia y utilizan pocos recursos.

\section{Método de Evaluación}

\subsection{Introducción}

El objetivo del método de evaluación es evaluar y medir el rendimiento de los procesos software de la organización para establecer rangos de eficiencia en base a "cómo funciona la organización con las mejores prácticas identificadas".

El método de evaluación propone una forma alternativa para evaluar el rendimiento de los procesos software de una organización al realizar: 1) un análisis de cobertura entre mejores prácticas internas, indicadores relacionados con los objetivos del negocio y objetivos del negocio, 2) un análisis de cumplimiento entre objetivos del negocio e indicadores relacionados; y 3) un análisis de priorización entre indicadores; en lugar de la aplicación de cuestionarios aplicados por los métodos de evaluación tradicionales. El método de evaluación propuesto se muestra en la Figura 3.

Como lo muestra la Figura 3, el método está formado por 7 actividades como sigue: 1) Analizar la cobertura de las mejores prácticas internas, indicadores y objetivos del negocio; 2) recoger valores planificados y reales de los indicadores; 3) realizar y llenar la matriz de cumplimiento; 4) establecer el rendimiento de los procesos; 5) realizar los gráficos de rendimiento; 6) analizar y priorizar los indicadores y; 7) comunicar los resultados. Las actividades serán descritas en secciones posteriores. 


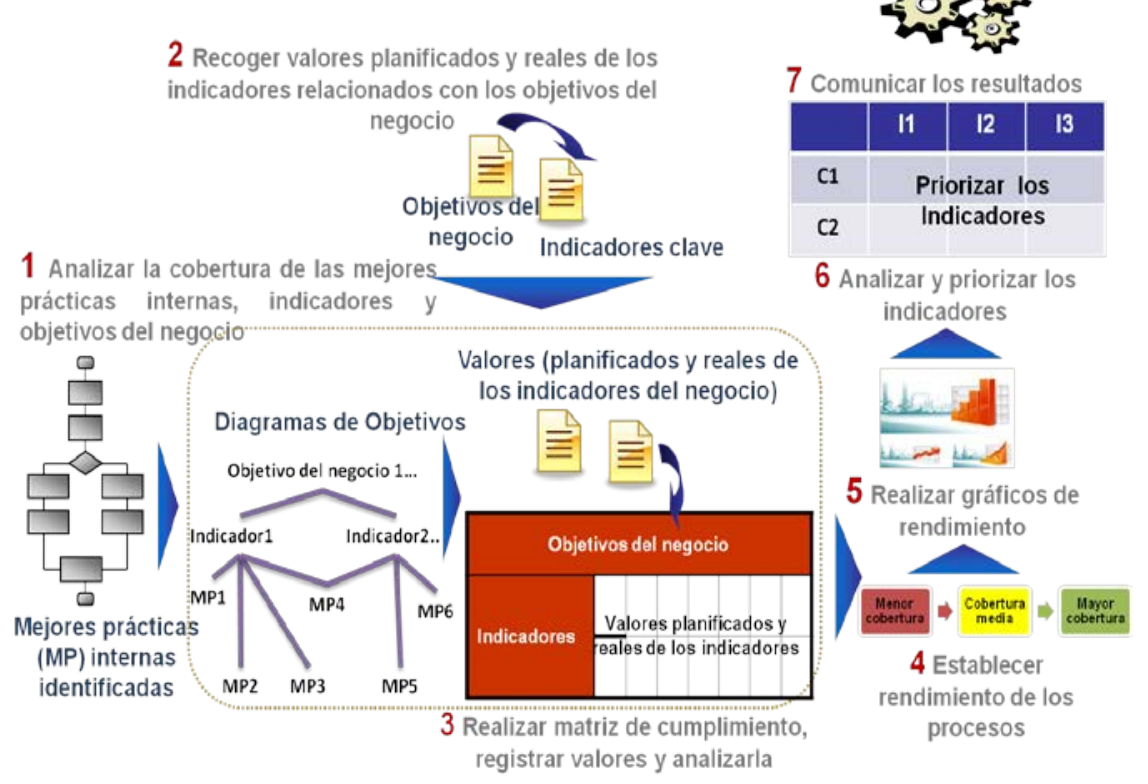

Figura 3. Método para evaluar rendimiento de los procesos

\subsection{Prerrequisito Para la Ejecución del Método de Evaluación}

Previo a la ejecución del método de evaluación, las mejores prácticas de la organización deben ser identificadas, para lo cual, se ejecuta el método para identificación de mejores prácticas, desarrollado como primera fase de la metodología MIGME-RRC.

La explicación del método para la identificación de mejores prácticas queda fuera del alcance de este artículo. Sin embargo, la Figura 4 muestra una visión general del mismo. Más información del método en[5][6].

El método de identificación de mejores prácticas propuesto se muestra en la Figura 4.

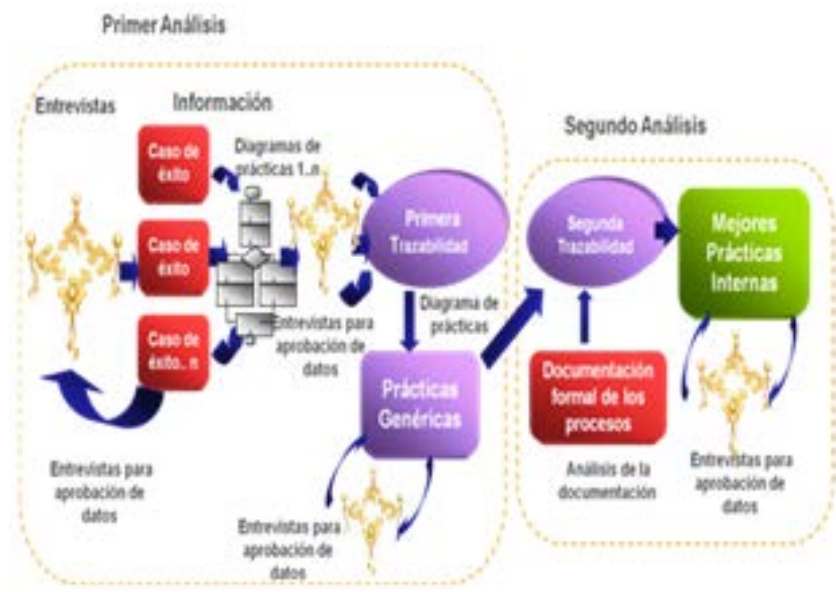

Figura 4. Método de identificación de mejores prácticas

Para tener una mejor comprensión de cómo es la interacción entre ambos métodos, en la Figura 5, se esquematiza la relación entre el método de identificación de mejores prácticas y el método de evaluación del rendimiento de los procesos. Además, se muestra la implicación de la alta dirección, mandos intermedios y usuarios de los procesos que logran ambos métodos.

Cómo se observa en la Figura 5 durante la ejecución del método para identificar mejores prácticas, los mandos intermedios y los usuarios de los procesos tienen una importante participación, debido a que son la fuente de extracción del conocimiento y validación de la formalización del mismo. Sin embargo una vez identificadas las mejores prácticas la alta dirección debe ser informada.

En el método de evaluación, la alta dirección tiene una importante participación, ya que es donde se definen los objetivos del negocio y se establecen los valores planificados para estos objetivos. Además, es donde se tiene acceso a la información de los valores reales de los indicadores y, son los encargados directos de decidir sobre los criterios de priorización de los indicadores.

Una vez que se ha establecido el rendimiento de los procesos y se han priorizado los indicadores, los resultados deben ser comunicados a los mandos intermedios y los usuarios de los procesos para sembrar la necesidad de la mejora de los procesos para alcanzar los objetivos del negocio establecidos.

Mediante la interacción lograda entre la alta dirección, mandos intermedios y usuarios de los procesos se logra la disminución de la resistencia al cambio, ya que los usuarios finales son los que proveen la entrada para la evaluación de los procesos, sin embargo, no tienen una implicación directa con el método de evaluación, por lo que en ningún momento se sienten que su trabajo se está evaluando. Sin embargo, al final del método se les proporciona información crítica para hacer crecer en ellos la necesidad de mejorar ya que se les proporciona: 1) el rendimiento de la organización con las prácticas llevadas a cabo (mejores prácticas de la organización), 2) se resalta la necesidad de mejorar para alcanzar los objetivos del negocio, y 3) hacia donde tiene que ser dirigido el esfuerzo de mejora 
Método para identificar mejores prácticas

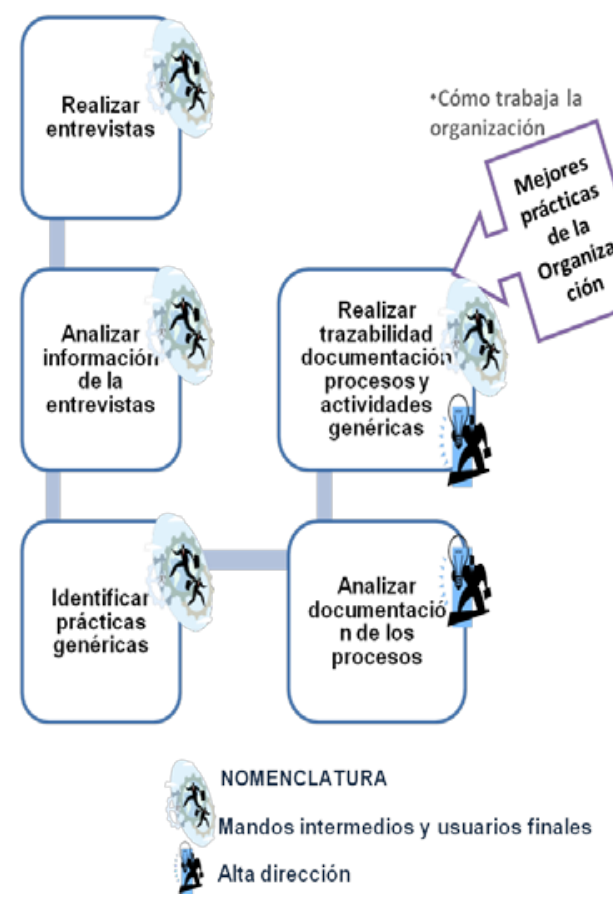

Figura 5. Interacción de métodos e implicados

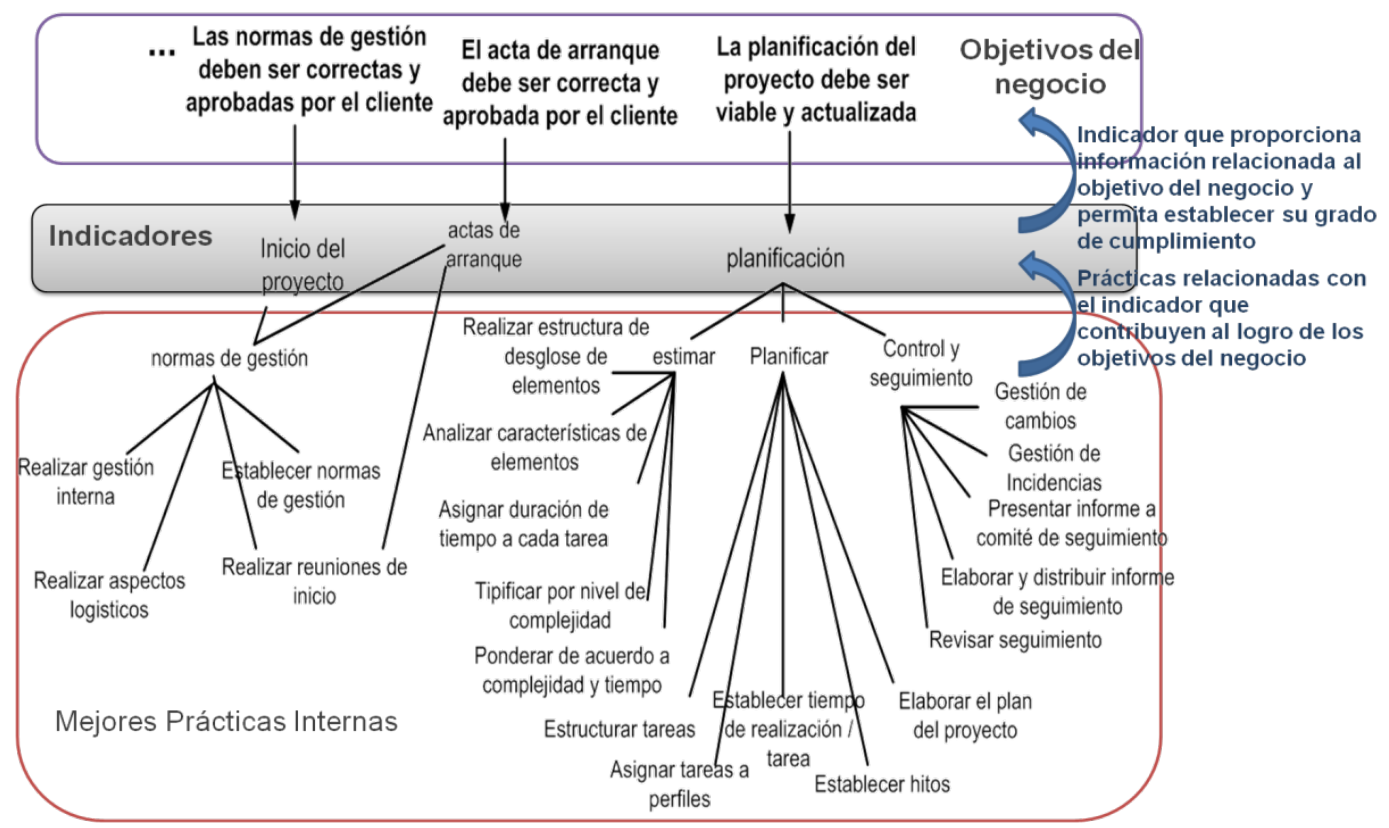

Figura 6. Diagrama de objetivos del negocio

\subsection{Descripción de Actividades del Método de Evaluación}

En esta sección se describen las actividades del método de evaluación y cómo fueron aplicadas en la práctica para realizar una evaluación interna rápida de los procesos de gestión de proyectos de una organización.

Cómo información adicional se considera importante resaltar que everis (http://www.everis.com/) es una empresa multinacional con presencia en Europa y América Latina. everis ofrece servicios para proporcionar soluciones a grandes compañías de cualquier sector y se basa en tres pilares: innovación y eficiencia (mediante la utilización de especialistas y metodologías, que hacen uso del conocimiento específico para cada proyecto), y productividad (al optimizar los resultados en tiempo y coste).

Desde su creación en 1996, ha crecido tanto en ingresos como en personal de una manera estable y orgánica. El volumen de su negocio para el 2009 fue de 404m€, tiene más de 7000 empleados y más de 1000 proyectos abiertos por 
mes.

A continuación se describen brevemente las actividades del método de evaluación, incluyendo los productos a obtener, la técnica utilizada y mostrando cómo ha sido su aplicación práctica en una empresa real.

\subsubsection{Analizar la Cobertura de las Mejores PrÁCticas Internas, Indicadores y Objetivos del Negocio}

El propósito de esta actividad es identificar las correspondencias entre las mejores prácticas identificadas, los indicadores y los objetivos del negocio mediante la realización de trazabilidad "bottom-up". Cobertura por lo tanto, es el grado de correspondencias que existe entre mejores prácticas, indicadores y objetivos del negocio.

Productos a obtener: diagrama de correspondencia de las mejores prácticas.

Técnica utilizada: diagramas de objetivos y reuniones de trabajo.

Aplicación práctica: la Figura 6 muestra parte del diagrama de 3 objetivos del negocio analizados en everis.

\subsubsection{Recoger Valores Planificados y Reales de los Indicadores}

- Valores planificados: la alta dirección tiene establecidos objetivos del negocio específicos a ser alcanzados. Como se ha mencionado anteriormente, cada objetivo del negocio tiene su indicador asociado que apoya a las organizaciones a ser específicos sobre las medidas de las que se tiene que obtener información sobre el cumplimiento de los objetivos del negocio.

- Valores reales: la fuente de esta información son las auditorías internas llevadas cabo en la organización.

Productos a obtener: valores planificados y reales de los indicadores.

Técnica utilizada: reunión de trabajo.

Aplicación práctica: la información relacionada con los objetivos del negocio fue solicitada al gestor de calidad. El gestor de calidad proporcionó la información de las auditorías internas llevadas a cabo para monitorear sus proyectos cada mes. La Tabla 2 muestra los valores planificados y reales de los indicadores recopilados.

Tabla 2. Valores planificados y reales de los indicadores

\begin{tabular}{|c|c|c|c|}
\hline$\#$ & Indicadores & Plan & Real \\
\hline 1 & $\begin{array}{c}\text { Las normas de gestión deben ser correctas y } \\
\text { aprobadas por el cliente }\end{array}$ & $\leq 5 \%$ & $15.30 \%$ \\
\hline 2 & $\begin{array}{c}\text { La planificación del proyecto debe ser viable } \\
\text { y actualizada }\end{array}$ & $\leq 5 \%$ & $9.30 \%$ \\
\hline 3 & Indicador 3* & $\leq 10 \%$ & $15,30 \%$ \\
\hline 4 & Indicador 4* & $\leq 10 \%$ & $17,30 \%$ \\
\hline 5 & Indicador 5* & $\leq 10 \%$ & $8 \%$ \\
\hline 6 & $\begin{array}{c}\text { El acta de arranque debe ser correcta y } \\
\text { aprobada por el cliente }\end{array}$ & $\leq 5 \%$ & 15.30 \\
\hline
\end{tabular}

* Por cuestiones de confidencialidad, los nombres de los indicadores 3, 4 y 5 han sido omitidos

4.3.3. Realizar y Llenar la Matriz de Cumplimiento
El propósito de esta actividad es realizar y llenar la matriz de cumplimiento. La matriz registra los objetivos del negocio en las columnas e indicadores en las filas. Posteriormente la matriz se llena con los valores planificados y reales recopilados en la actividad anterior.

Productos a obtener: matriz de evaluación del cumplimiento.

Técnica utilizada: matriz de evaluación (cumplimiento) y reuniones de trabajo.

Aplicación práctica: se realizó la matriz de cumplimiento registrando los datos de los indicadores. En la Figura 7 se puede observar los objetivos del negocio en las columnas y los indicadores en las filas.

\subsubsection{Establecer el Rendimiento de los Procesos}

El propósito de esta actividad es establecer el cumplimiento de los indicadores mediante la comparación de los valores planificados y reales, mediante la aplicación de la fórmula:

Cumplimiento $=$ valor real - valor planificado

A través del análisis del porcentaje resultante se establece el grado de cumplimiento de los indicadores, y por lo tanto, de los objetivos del negocio.

Además, para realizar este análisis se propone utilizar una escala de colores para resaltar los valores resultantes de acuerdo al grado de cobertura de tal manera que:

- Indicadores que han resultado con un mayor porcentaje son resaltados con color anaranjado. Esto indica que el objetivo del negocio tiene una menor cobertura.

- Indicadores que han resultado con un menor porcentaje son resaltados con verde. Esto indica que el objetivo del negocio tiene una mayor cobertura.

- Indicadores que han resultado con un porcentaje medio son resaltados con amarillo. Esto indica que el objetivo tiene una cobertura media.

Productos a obtener: matriz de análisis del cumplimiento con indicadores clasificados.

Técnica utilizada: matriz de evaluación (cumplimiento) y reuniones de trabajo.

Aplicación práctica: la Figura 7 muestra el análisis del cumplimiento realizado entre objetivos del negocio e indicadores.

\begin{tabular}{|c|c|c|c|c|c|c|}
\hline \multirow[b]{2}{*}{ Indicadores } & \multicolumn{5}{|c|}{ Objetivos del negocio } & \multirow[b]{2}{*}{  } \\
\hline & 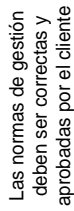 & 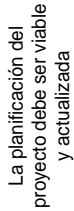 & 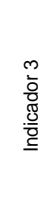 & 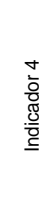 & $\begin{array}{l}\text { L } \\
\overline{0} \\
\frac{0}{0} \\
\text { 음 }\end{array}$ & \\
\hline $\begin{array}{l}\text { Normas de } \\
\text { gestión }\end{array}$ & $10,3 \%$ & & & & & \\
\hline $\begin{array}{l}\text { Planificación } \\
\text { del proyecto }\end{array}$ & & $4,3 \%$ & & & & \\
\hline Indicador 3 & & & $5,3 \%$ & & & \\
\hline Indicador 4 & & & & $7,3 \%$ & & \\
\hline Indicador 5 & & & & & $-2 \%$ & \\
\hline $\begin{array}{c}\text { Acta de } \\
\text { arranque }\end{array}$ & & & & & & $10,3 \%$ \\
\hline
\end{tabular}

Figura 7. Matriz de cumplimiento 


\subsubsection{Realizar los Gráficos de Rendimiento}

El propósito de esta actividad es realizar el o los gráficos que permitan una mejor comprensión del rendimiento de los procesos; estos gráficos permitirán resaltar la necesidad de la mejora de los procesos para el cumplimiento de los objetivos del negocio.

Productos a obtener: gráficos del rendimiento de los procesos.

Técnica utilizada: reuniones de trabajo.

Aplicación práctica: la Figura 8 muestra el gráfico realizado del rendimiento de los procesos.

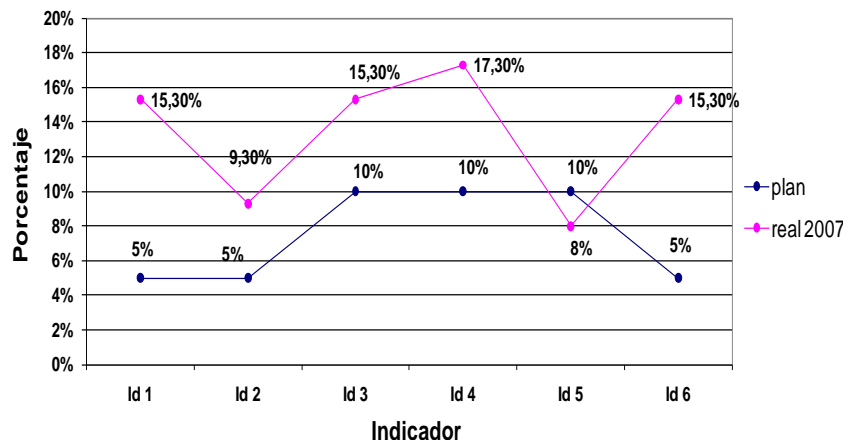

Figura 8. Cumplimiento de los indicadores

\subsubsection{Analizar y Priorizar los Indicadores}

El propósito de esta actividad es analizar los indicadores y elegir aquellos en los cuales se considere que existe una oportunidad de mejora y realizar una lista con estos indicadores.

Posteriormente, asignar pesos a los indicadores de acuerdo a los criterios propios de la organización o criterios como: ahorro de costes, incidencias de problemas de calidad, problemas de tiempo, problemas de respuestas, frustración del personal, quejas de clientes, viabilidad.

Los criterios deben ser elegidos de acuerdo a las necesidades de mejora de la organización. El peso asignado será asignado dentro de una escala de 5 (peso máximo) a 0 (peso mínimo).

Productos a obtener: lista de indicadores priorizados.

Técnica utilizada: reuniones de trabajo.

Aplicación práctica: la Tabla 3 muestra la priorización de los indicadores.

Tabla 3. Priorización de indicadores del negocio

\begin{tabular}{|c|c|}
\hline Indicadores & Peso \\
\hline Normas de gestión & 5 \\
\hline Planificación del proyecto & 5 \\
\hline Indicador 3 & 3 \\
\hline Indicador 4 & 2 \\
\hline Indicador 5 & 3 \\
\hline Actas de arranque & 5 \\
\hline
\end{tabular}

\subsubsection{Comunicar los Resultados}

El propósito de esta actividad es comunicar a los partes interesadas los resultados tanto del rendimiento de los procesos como de la priorización de los indicadores del negocio a ser mejorados.
Productos a obtener: material para la presentación de los resultados

Técnica utilizada: reuniones de trabajo.

Aplicación práctica: se dieron a conocer los resultados del análisis del rendimiento de los procesos con las prácticas existentes. Para lograrlo, se mantuvieron reuniones con todo el personal implicado con el proyecto de mejora. El objetivo de estas reuniones fue resaltar la necesidad para mejorar los procesos, la importancia de la participación de los implicados para el éxito de la mejora y hacia donde se tenían que dirigir los esfuerzos de mejora. La reunión se apoya con diapositivas en las que se muestran los gráficos con los resultados del análisis.

\section{Resultados}

Es importante resaltar que este método ha sido aplicado como la segunda fase de la metodología MIGME-RRC.

En esta sección se muestran los resultados del cumplimiento de los indicadores, posterior a la implementación de la mejora de procesos.

La Figura 9 muestra las gráficas de control del cumplimiento de los indicadores después de la mejora. Como se observa en las tres gráficas existe una disminución en la media y en los valores de los límites superior e inferior entre datos antes y durante la mejora (grupo I) y después de la mejora (Grupo II).

Aplicación práctica: la Figura 9 muestra el gráfico realizado del rendimiento de los procesos.

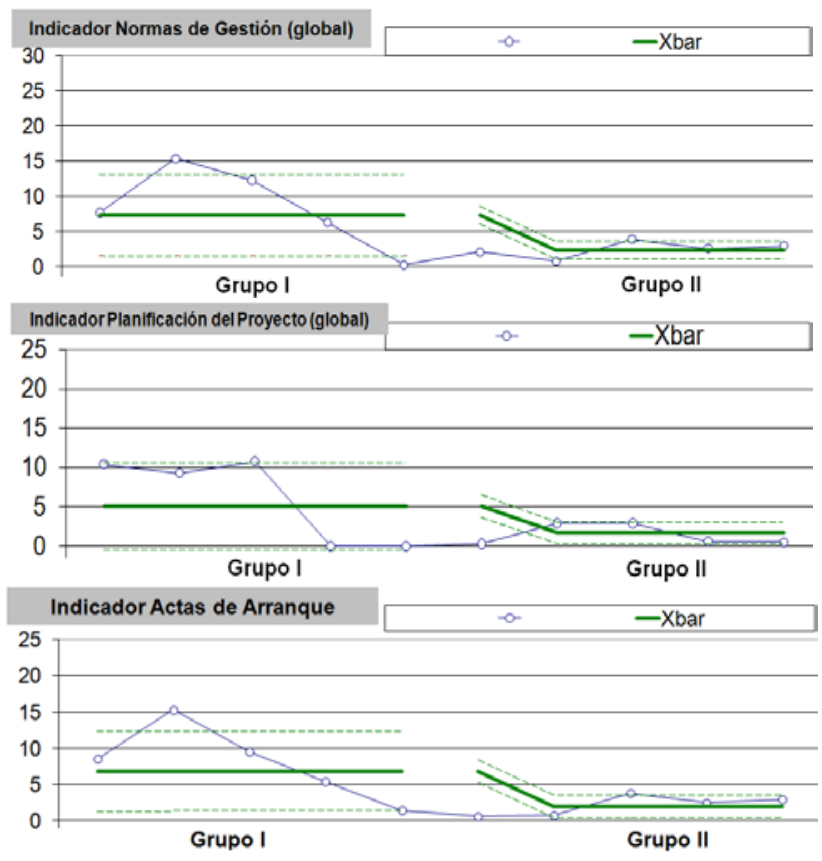

Figura 9. Análisis de los indicadores posterior a la mejora

\section{Conclusiones}

La aplicación del método de evaluación ha permitido realizar una evaluación rápida del rendimiento de los 
procesos de gestión de proyectos de la organización. Con los resultados obtenidos, se ha podido dirigir el esfuerzo dedicado a la mejora hacia aquellos procesos que necesitaban mejorarse de acuerdo a las necesidades de sus objetivos del negocio y se ha logrado obtener un mayor compromiso tanto de la alta dirección como los mandos intermedios y usuarios de los procesos.

Además se considera importante resaltar que por la naturaleza del método este puede ser fácilmente implementado en cualquier organización (grande o PyME) que desee conocer hacia dónde dirigir su es esfuerzo de mejora.

Como trabajo futuro se está investigando sobre el desarrollo de herramientas y/o plataformas que apoyen en la implementación de los métodos que conforman la metodología MIGME-RRC, así como extender la aplicación de la metodología hacia otros ramos industriales.

Los resultados muestran que llevar a cabo la evaluación mediante el uso de este método ha permitido llevar a cabo una mejora de procesos exitosa.

\section{AGRADECIMIENTOS}

Este trabajo ha sido patrocinado por el Centro de Investigación en Matemáticas (CIMAT) unidad Zacatecas, everis Foundation y la Universidad Politécnica de Madrid a través de la "Cátedra de Mejora del Proceso Software en el Espacio Iberoamericano”..

\section{REFERENCIAS}

[1] Mishra D., Mishra A. Software Process Improvement in SMEs: A Comparative View. Computer Science and Information Systems, Vol. 6, No. 1, 111-140. (2009).

[2] Gupta J., Sharma S., Hsu, J. An overview of knowledge management. Idea Group Inc.Ch. 1. (2004).

[3] Molina J.L., Marsal M. La gestión del conocimiento en las organizaciones, pp. 60-68, 87-94. (2002).

[4] Turban E., Aronson J.E., Liang T-P. Knowledge Management. Decision Support Systems and Intelligent Systems, Pearson (Prentice Hall), ch. 9, pp. 487. (2005).

[5] Calvo-Manzano Jose A., Cuevas Gonzalo, Mejia Jezreel, Muñoz Mirna, San Feliu Tomás, Sánchez Angel, Rocha Alvaro. "Approach to Identify Iternal Best Practices in a Software Organization”, System, Software and Services Process Improvement, 17th European Conference proceedings, ISSN 1865-0929, EuroSPI 2010, Grenoble, France, (2010).

[6] Calvo Manzano J. A., Cuevas G., Muñoz M. A., San Feliu T.,
Álvaro Rocha, Sánchez A., "Identificación de las Mejores Prácticas de una Organización de Desarrollo de Software Mediante la Gestión de su Conocimiento”, Proceedings de la $5^{\text {a }}$ Conferencia Ibérica de Sistemas y Tecnologías de la Información CISTI 2010, ISBN: 978-989-96247-2-6. (2010).

[7] Pino F.P., García F., Piattini M., Herramienta de Soporte a la Valoración Rápida de Procesos Software, IEEE Latin America Transactions, Vol. 5, No. 4, July 2007, pp 218-223. (2007).

[8] Garcia I., Pacheco C., Cruz D. Adopting a RIA-based tool for supporting assessment, implementation and learning in software in software process improvement under the NMX-I-059/02-NYCE-2005 standard in small software enterprises. Eighth ACIS International Conference on Software Engineering Research, Management and Applications. (2010).

[9] Pino F.J., Pardo C., García F., Piattini M. Assessment methodology for software process improvement in small organizations" Inf.Softw.Technol. vol. 52, pp.1044-1061. (2010).

[10] CMMI Working group: The Economics of CMMI®, NDIA Systems Engineering Division,Version 1.0. (2009).

[11] Withers D.H.: Software engineering best practices applied to the modeling process. In: Simulation Conference Proceedings, 2000. Winter, pp. 432-439 vol.1. (2000).

[12] Brotbeck G., Miller T., Statz J.: A survey of Current Best Practices and Utilization of Standards In the Public and Private Sectors. Department of Information Resources. (2006).

[13] Chrissis M.B., Konrad M., Shrum S.: CMMI Second Edition Guidelines for Process Integration and Product Improvement, United States, Massachusetts, (eds): Addison Wesley. (2007).

[14] Humphrey W.: Introduction to the Team Software Process, Massachusetts, (eds.) Addison-Wesley, pp. 459. (2006).

[15] IEEE Computer Society: IEEE Guide Adoption of PMI Standard A Guide to the Project Management Body of Knowledge; IEEE Std 1490-2003 (Revision of IEEE Std 1490-1998), pp. 0_1-216. (2004).

[16] International Organization for Standardization: ISO/IEC 15504: 2004 Information technology - Process assessment. (2004).

[17] Standard Comitee IEEE 2008. "Systems and Software Engineering-Software Life Cycle Processes. IEEE STD 12207-2008." ieeexplorer: cl 138. (2008).

[18] Goethert W., Hayes W., Experiences in Implementing Measurement Programs, Software Engineering Institute, Technical Note CMU/SEI-2001-TN-026. (2001).

[19] Calvo-Manzano J.A., Cuevas G., Gómez G., Mejia J., Muñoz M., San Feliu T. Methodology for process improvement through basic components and focusing on the resistance to change. Journal of Software Maintenance and Evolution: Research and Practice. (2010). 